\title{
A Case Study on Computer-Based Analysis of the Stochastic Stability of Mechanical Structures Driven by White and Colored Noise: Utilizing Artificial Intelligence Techniques to Design an Effective Active Suspension System
}

\author{
Aydin Azizi $(\mathbb{D}$ \\ School of Engineering, Computing and Mathematics, Oxford Brookes University, Wheatley Campus, Oxford No. 33 1HX, UK \\ Correspondence should be addressed to Aydin Azizi; aydin.azizi@brookes.ac.uk
}

Received 16 April 2019; Revised 6 November 2019; Accepted 29 January 2020; Published 25 April 2020

Academic Editor: András Rontó

Copyright ( 2020 Aydin Azizi. This is an open access article distributed under the Creative Commons Attribution License, which permits unrestricted use, distribution, and reproduction in any medium, provided the original work is properly cited.

\begin{abstract}
The goal of this research is to design an Artificial Intelligence controller for the active suspension system of vehicles. The Ring Probabilistic Logic Neural Network (RPLNN) architecture has been adopted to design the proposed controller, and the pavement condition has been modelled utilizing Gaussian white noise. The results show that the proposed RPLNN controller has an effective performance to reduce the unwanted stochastic effect of the road profile.
\end{abstract}

\section{Introduction}

1.1. Background. During the car transportation due to conversion of absorbed energy by suspension and tire of the vehicle to thermal energy, an energy loss happens. Utilizing an effective suspension system gives an opportunity to reduce the energy loss and save energy [1], and saving energy means reducing the fuel consumption. In recent years, many researchers focus on reducing the undesirable effects of the pavement and decrease the fuel consumption rate by designing effective suspension systems [2].

In 2012, Ismail et al. [3] proposed an Artificial Neural Network (ANN) model for a light-duty diesel engine powered using blends of various biodiesel fuels with conventional fossil diesel. Cay et al. [4] utilized ANN to predict the brake specific fuel consumption. In 2014, Roy et al. [5] modelled the performance and emission parameters of a single-cylinder four-stroke engine under CNG-diesel dual-fuel mode by ANN. In 2015, the performance, emission, and combustion characteristics of a single-cylinder, four-stroke variable compression ratio engine were predicted with the aid of ANN by Muralidharan and Vasudevan [6]. As an intelligent technique, particle swarm optimization has been adopted by Olivera et al. [7] to reduce vehicle emissions and fuel consumption in the city. In 2016, Lotfan et al. [8] combined ANN and nondominated sorting genetic algorithm II to model and reduce $\mathrm{CO}$ and NOx emissions from a direct injection dual-fuel engine. ANN has been utilized by Perrotta et al. [9] in 2017 to model fuel consumption of trucks. In 2018, Azizi [10] proposed a PID controller to control fuel consumption rate based on the changing pavement condition. Although the result of the research shows that the proposed PID controller can reduce the fuel consumption, but the vibration at the beginning of the control procedure because of the input control signal for few seconds is high.

The current research aims to design an effective Artificial Intelligence (AI) architecture to decrease the effect of the road profile which shows itself as imposed vibration on vehicle. It means less fuel consumption and higher parts life can be achieved by filtering such a noise. In this research, Gaussian white noise has been adopted to model the road profile. Different from previous works, the aim of the current research is presenting an effective approach to eliminate the mentioned fluctuation and 
control the fuel consumption rate even at the beginning of the control process $[11,12]$. For this purpose, Ring Probabilistic Logic Neural Network (RPLNN) which is an effective paradigm and has been utilized by researchers as a function optimizer, prediction tool, and plant simulator [13-16] is adopted here to be trained by the PID controller proposed by Azizi et al. [10]. In this paper, MATLAB software and Simulink toolbox have been utilized to develop a simulation-based model of the active suspension system of the vehicle and design an effective RPLNN controller to investigate and reduce the effect of the imposed vibration from the road profile. The proposed simulator is capable of simulating the vertical displacement of the vehicle before and after utilizing the AI controller, also it is capable of analyzing these vibrations by examining the different values of the system parameters. The results show that the proposed RPLNN controller has a superior performance than the PID controller and has an effective performance to reduce the imposed vibration by the road profile and reducing the fuel consumption rate.

After the brief introduction and literature review about the research topic, the remaining parts of the paper is organized in three more sections. First, in Section 2, mathematical modeling of a proposed active suspension of a quarter car model proposed RPLNN algorithm, and the simulation-based model set up have been discussed in detail. In Section 3, which is the results section, the designed simulator has been verified by analyzing the effect of the proposed controller's effect on system vertical displacement. Finally, in Section 4, the summary of the research has been given and possible future works have been suggested.

\section{Methodology}

Fuel consumption rate can be affected by two important factors: vehicle handling performance and vehicle suspension system [17]. In conventional method, a passive suspension system which is a combination of springs and dampers has been utilized to reduce the imposed vibrations to the vehicle from the pavement profile. It is important to know that, since the damping ratio cannot be adjusted, so such a system is not able to eliminate the imposed vibration. Nowadays, by advancement in technology active suspension systems which are equipped with effective controllers are being utilized to reduce the effect of pavement conditions. [18].

The ultimate goal of this research has been defined as to design an effective AI controller to reduce the undesirable effect of the road profile on vehicles. The steps of the proposed methodology in this research have been defined in the following order. The first step is introducing the quarter car model equipped with an active suspension system. The second step is utlizing RPLNN algorithm to design an efficient controllerlas a part of the active suspension system. The third step is utilizing MATLAB software to train the proposed AI controller. The next step is simulating the proposed set up utilizing Simulink, and the last step is testing and analyzing the performance of the proposed RPLNN controller and comparing it with the performance of the PID controller.

2.1. Active Suspension Controller Model. The quarter car model, which is a model to investigate the dynamic vibration of a vehicle with regards to the road profile as the input of the model, has been illustrated in Figure 1. It is important to know that, in such a model some parameters such as geometrical information, pitching, and rolling angles have not been neglected, but most of the important features such as the change of the load has been considered.

In the current work, to generate the mathematical model, the state space method which is one of the well-known approaches in control engineering has been adopted [20].

To generate the state space representation, the first step is analyzing the quarter car model (Figure 1), which has been carried out by the following equations:

$$
\begin{aligned}
& M_{1} \ddot{x}_{1}(t)+D\left[\dot{x}_{1}(t)-\dot{x}_{2}(t)\right]+k 1\left[x_{1}(t)-x_{2}(t)\right]=u, \\
& M_{2} \ddot{x}_{2}(t)-D\left[\dot{x}_{1}(t)-\dot{x}_{2}(t)\right]+k_{1}\left[x_{2}(t)-x_{1}(t)\right] \\
& \quad+k_{2}\left[x_{2}(t)-x_{0}(t)\right]=-u .
\end{aligned}
$$

$M_{1}(300 \mathrm{~kg})$ and $M_{2}(40 \mathrm{~kg})$ are the sprung and unsprung masses, respectively. $K_{1}(15000 \mathrm{~N} / \mathrm{m})$ and $D$ $(1000 \mathrm{~N} / \mathrm{m})$ are stiffness and damping coefficients of spring and damper of the suspension system, respectively. $K_{2}$ $(150000 \mathrm{~N} / \mathrm{m})$ is the stiffness coefficient of the spring which is the model of the tire of the vehicle. The vertical displacements of the sprung mass, unsprung mass, and pavement condition have been shown by $X_{1}(t), X_{2}(t)$, and $X_{0}(t)$, respectively, and the parameter $\mathrm{U}$ represents the controlled force.

Now, the state variables with respect to the dynamic equations (1) and (2) and can be defined as follows:

$$
\begin{aligned}
& x_{1}=x_{2}(t), \\
& x_{2}=x_{1}(t), \\
& x_{3}=\dot{x}_{2}(t), \\
& x_{4}=\dot{x}_{1}(t) .
\end{aligned}
$$

By substituting state variables presented on equation (3) in equations (2) and (3), we will get the following expressions:

$$
\begin{aligned}
& \dot{x}_{4}=-\frac{D}{M_{1}} x_{4}+\frac{D}{M_{1}} x_{3}-\frac{K_{1}}{M_{1}} x_{2}+\frac{K_{1}}{M_{1}} x_{1}+\frac{u}{M_{1}}, \\
& \dot{x}_{3}=\frac{D}{M_{2}} x_{4}-\frac{D}{M_{2}} x_{3}-\frac{K_{1}}{M_{2}} x_{1}+\frac{K_{1}}{M_{2}} x_{2}-\frac{K_{2}}{M_{2}} x_{1}+\frac{K_{2}}{M_{2}} x_{0}-\frac{u}{M_{2}},
\end{aligned}
$$

In general state space variables, change can be defined as follows: 


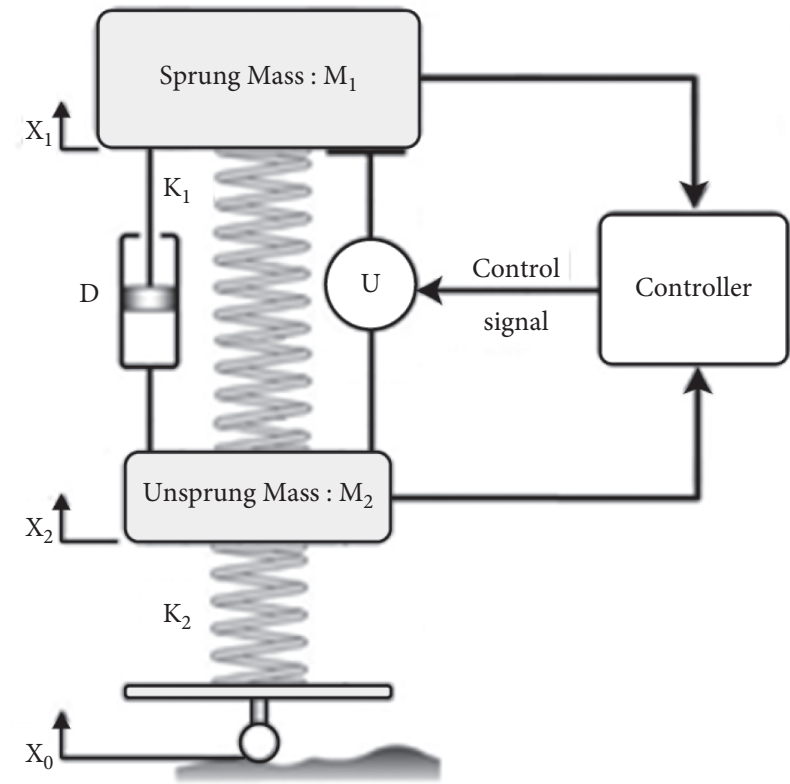

FIGURE 1: Quarter car model equipped with the active suspension system [19].

$$
\begin{aligned}
\frac{\mathrm{d} X}{\mathrm{~d} t} & =\mathrm{AX}+\mathrm{BU}, \\
X & =\left[\begin{array}{llll}
x_{1} & x_{2} & x_{3} & x_{4}
\end{array}\right]^{T} .
\end{aligned}
$$

So, based on equations (4) and (5) matrixes A and B of equation (6) will be as follows:

$$
\begin{gathered}
A=\left[\begin{array}{cccc}
0 & 0 & 1 & 0 \\
0 & 0 & 0 & 1 \\
-\frac{k_{1}+k_{2}}{M_{2}} & \frac{k_{1}}{M_{2}} & -\frac{D}{M_{2}} & \frac{D}{M_{2}} \\
B & =\left[\begin{array}{cccc}
\frac{k_{1}}{M_{1}} & -\frac{k_{1}}{M_{1}} & \frac{D}{M_{1}} & -\frac{D}{M_{1}}
\end{array}\right], \\
0 & 0 \\
\frac{k_{2}}{M_{2}} & -\frac{1}{M_{2}} \\
0 & \frac{1}{M_{1}}
\end{array}\right] .
\end{gathered}
$$

The system input variable matrix is

$$
U=\left[\begin{array}{ll}
x_{0}(t) & u
\end{array}\right]^{T} \text {. }
$$

The vehicle suspension system output matrix equation is

$$
Y=\mathrm{CX}+\mathrm{DU}
$$

In this paper, the output has been defined as the difference of the vertical displacement of the sprung and unsprung masses:

$$
Y=x_{2}(t)-x_{1}(t)
$$

So, based on equation (11) matrixes $C$ and $D$ of equation (10) will be as follows:

$$
\begin{aligned}
& C=\left[\begin{array}{llll}
-1 & 1 & 0 & 0
\end{array}\right], \\
& D=\left[\begin{array}{ll}
0 & 0
\end{array}\right] .
\end{aligned}
$$

After introducing the mathematical model of the quarter car model, the next step is investigation of one of the inputs of this mathematical model which is the pavement condition.

2.2. White Noise Road Input Signal. Mathematical modeling of the pavement condition due to its Gauss-distributed stochastic behavior is too hard. To fulfill this task, the Gaussian white noise which shows randomness behavior can be utilized as the representative of the statistical pavement power spectral density. As shown in equation (13), the relationship of the random road input signal and the Gaussian white noise per ISO/TC108/SC2N67 can be modelled mathematically as shown in the following equation [21]:

$$
\frac{q(s)}{w(s)}=\frac{2 \pi n_{0} \sqrt{G_{q}\left(n_{0}\right) v}}{s+2 \pi f_{0}} \text {. }
$$

where $q(s)$ is the random road input signal, $w(t)$ is the Gaussian white noise, $n_{0}$ is Reference Spatial frequency, $G_{\mathrm{q}}$ $\left(n_{0}\right)$ is the Road roughness coefficient, and $f_{0}$ is the Filter lower-cut-off frequency.

After introducing the quarter car mathematical model and the Gaussian white noise as one the input signals of it, the next step is the design of the controller utilizing the RPLNN algorithm.

2.3. Ring Probabilistic Logic Neural Networks Controller. Probabilistic Logic Neuron (PLN) is a weightless neuron which its output can be defined as one, zero, or "don't care" states [22]. Because of the "don't care" state, the PLNs have flexibility to bind together and design a fast-learning weightless neural networks [14]. Based on this property the RPLNN has been proposed by Menhaj et al. [23] as a novel algorithm to model, control, and optimize complex plants. The proposed design consists of PLNs in which the output of the last one is fed by the input of the first one (see Figure 2).

In 2016, Azizi et al. $[15,22]$ utilized RPLNN structure as an optimizer tool to optimize weightless neural networks and RFID networks. Later, the RPLNN structure has been developed and utilized as a part of the hybrid optimizers $[13,14,16,24,25]$.

In the current research, different from previous works, the weightless RPLNN has been utilized to be trained by the 


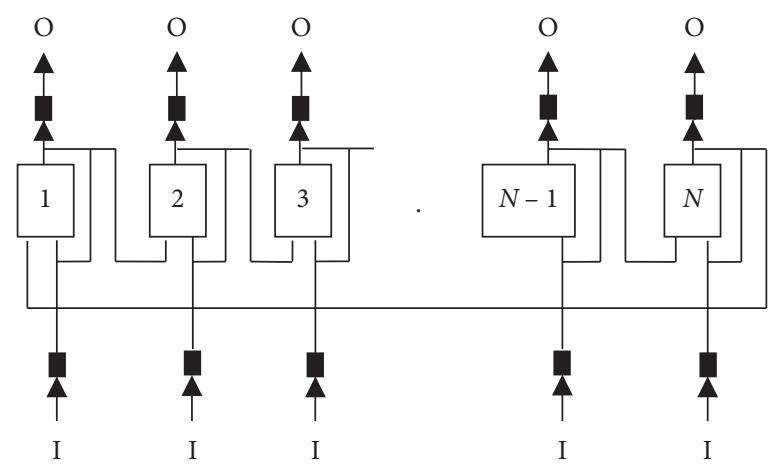

FIGURE 2: RPLNN structure [13].

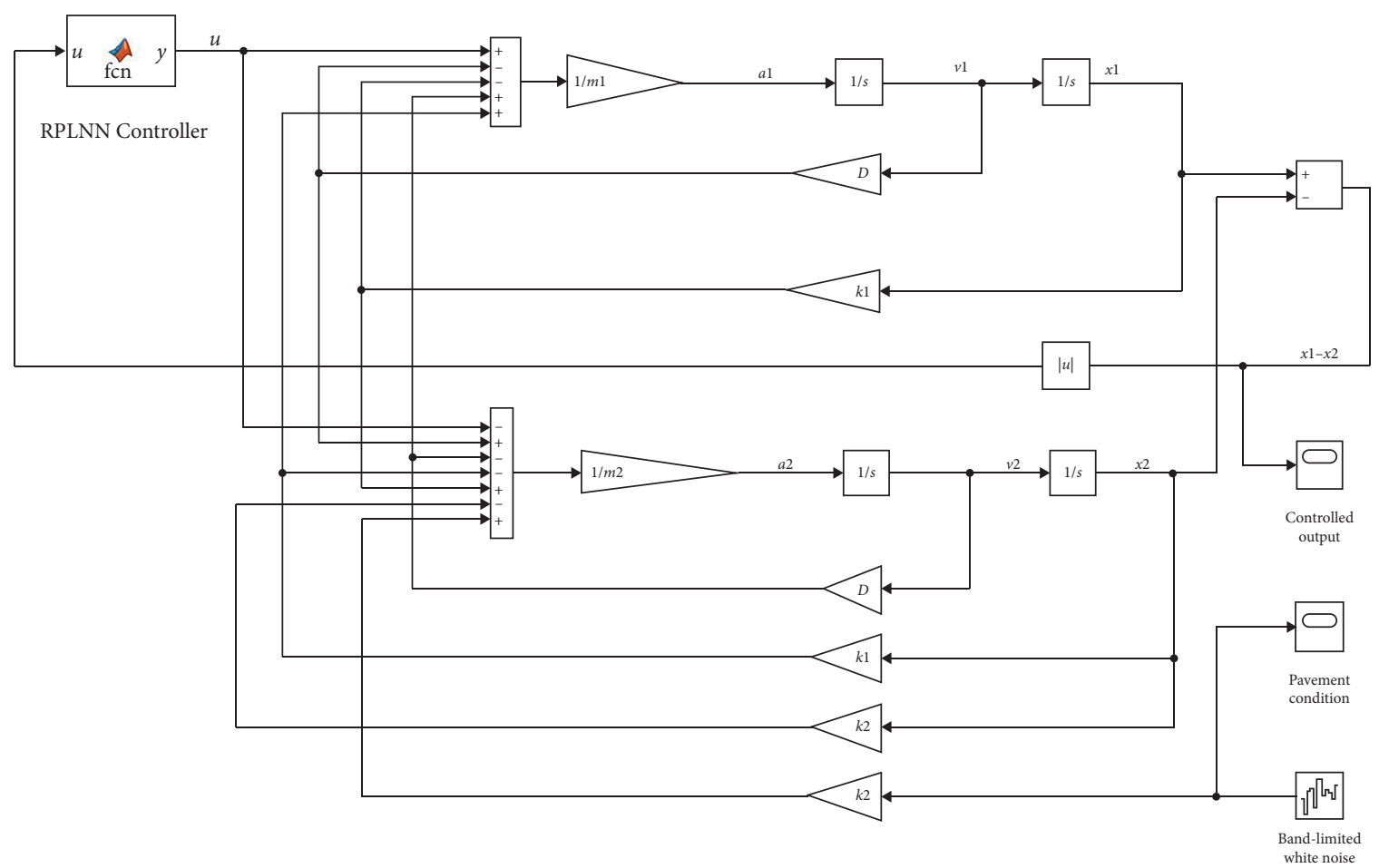

Figure 3: MATLAB Simulink Model of the Noise Cancellation system for the quarter car model.

proposed PID controller in previous reserach [10], so after the accomplishment of the training process, the proposed RPLNN has been utilized as the controller to reduce the vertical vibration of the vehicle due to the road conditions which can be considered as Gaussian white noise input.

The input of the RPLNN will be the vertical displacement of the vehicle, and the output will be the actuator's force. It means that the proposed controller regulates the active suspension system's force considering the vibration due to road condition.

To fulfill this task, it is essential to define an objective function. Since the proposed algorithm gives a fitter result in each iteration, so it can be called as an evolutionary algorithm. Here, since the aim is reducing the difference between the vertical displacements of the sprung and unsprung masses, so the objective function can be defined as the error function (see equation (14)). In this case by minimizing the objective function the RPLNN controller will be able to adjust the active suspension force. It is important to know that to train the RPLNN controller the reference modelis the data of the PID controller, which has been proposed by Azizi at el. $[10,26]$ :

$$
E(t)=x_{1}(t)-x_{2}(t)
$$

\section{Results}

As seen in Figure 3, the proposed quarter car model has two inputs: the first one is the road condition which is taken as random input and has been modelled by Gaussian white noise function, and the second input is the generated force by the RPLNN controller to reduce the effect of the imposed noise to the vehicle. 


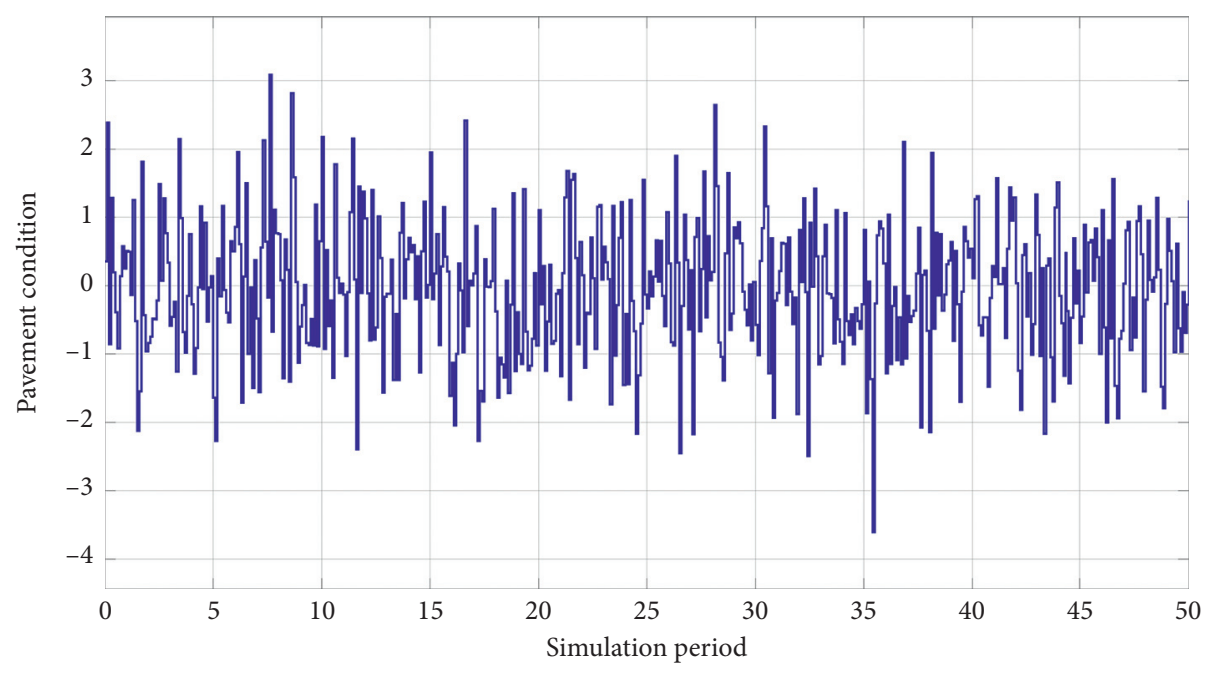

— Pavement condition

Figure 4: Pavement condition.

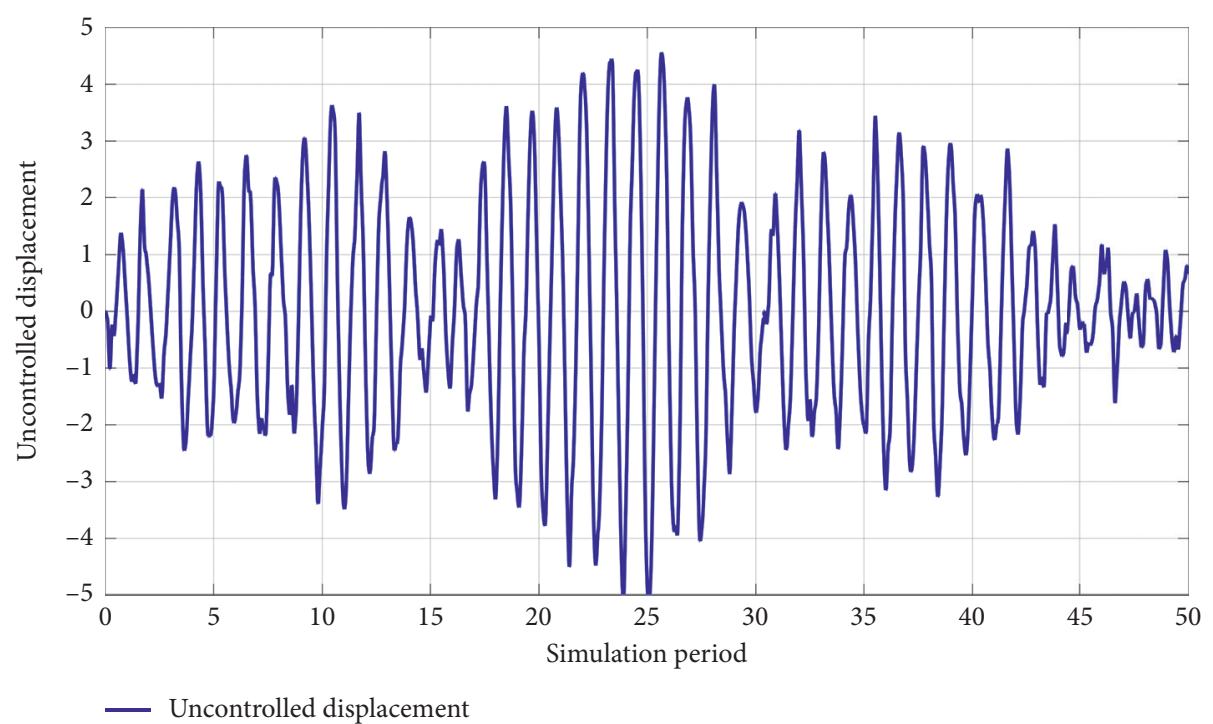

Figure 5: Uncontrolled displacement.

As seen in Figure 4, first input, which is the road condition, has been modelled by Gaussian white noise and the effect of this noise, which is the vertical displacement of the car, has been shown as uncontrolled displacement in Figure 5 .

As seen in Figure 5, the quarter car model fluctuates based on the road input signal. Since it is the uncontrolled behavior, so by comparing Figures 4 and 5, it can be observed that the vehicle completely follows the road condition which can results in increasing the fuel consumption and compromising passenger's safety.

As it is mentioned previously, the reference model which has been utilized to train the RPLNN is the data of the PID controller which has been presented in Figures 6 and 7 .

The RPLNN controller has been trained offline by running the training for 100 iterations. As seen in Figure 8, at the end of the training procedure the error function value has been reduced to 0.001 . The output of the systemby utilizing RPLNN controller has been calculated and shown in Figure 9.

By comparing Figures 5 and 9, which are the indicators of the behavior of the system equipped and not equipped with RPLNN controller, it can be observed that the system equipped with the RPLNN controller reduces the effect of the pavement on the vehicle and reduces the vibrations.

Noise cancelation results utilizing PID and RPLNN controllers have been shown in Figures 9 and 10, respectively. By comparing these two figures, it can be observed that both controllers are able to reduce the undesired effect of the pavement profile on the vehicle. It is important to mention that although both controllers are able to reduce the vertical vibrations in the same simulation period, but since the active suspension system equipped with the RPLNN 


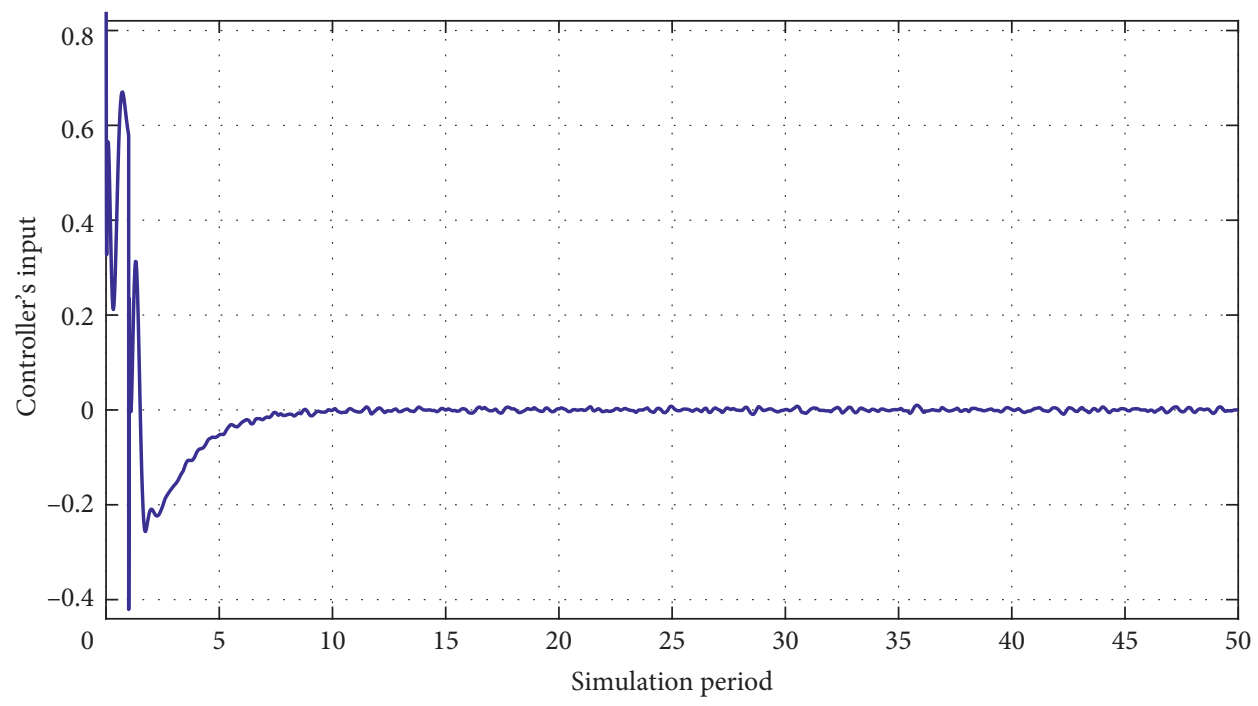

— Controller's input

FIgURE 6: Reference Input data (PID controller) of the controller.

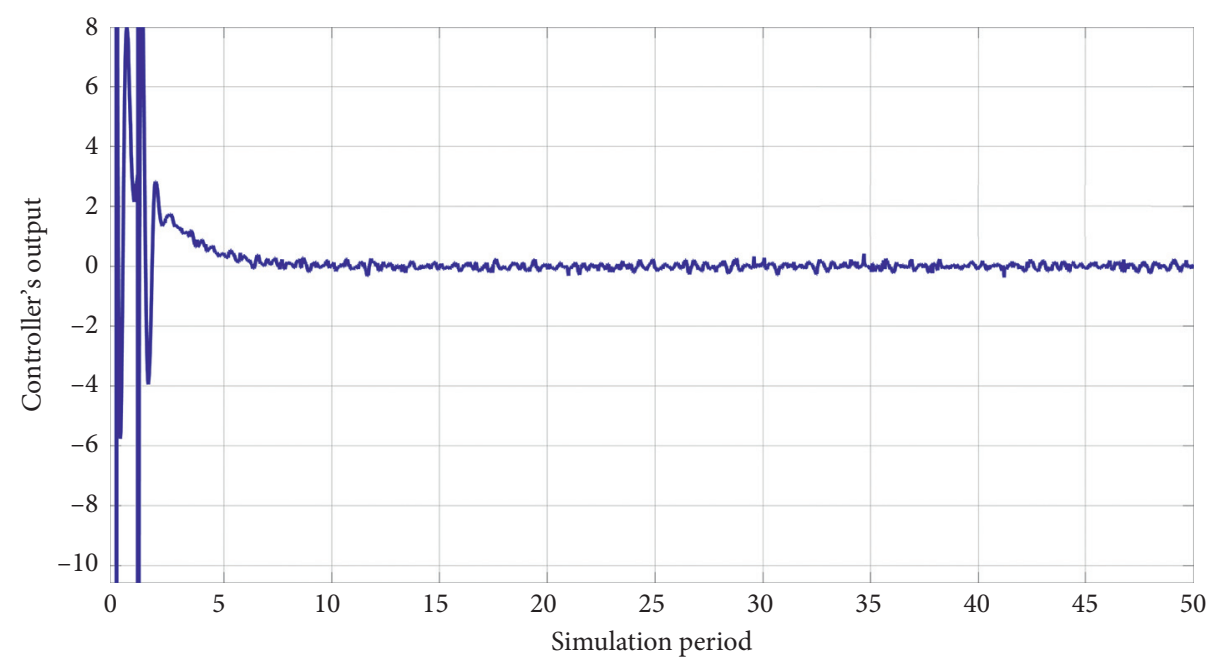

— Controller's output

FIGURE 7: Reference output data (PID controller) of the controller.

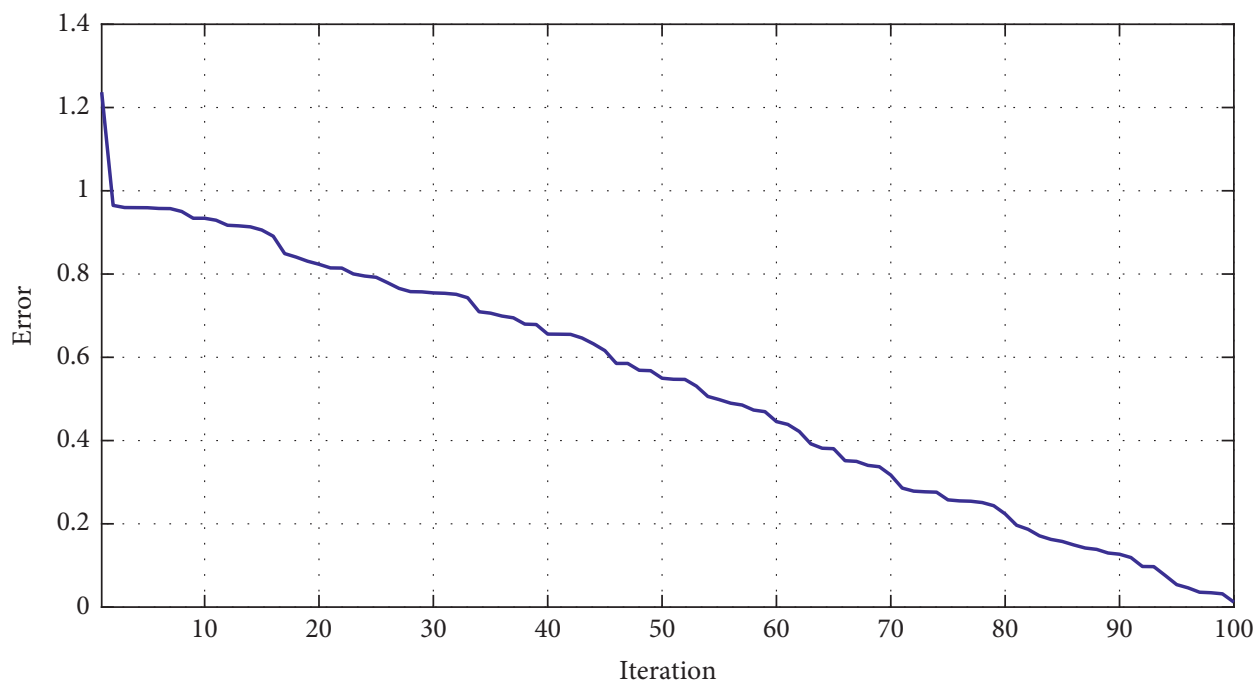

- Error

Figure 8: Error in each training iteration. 


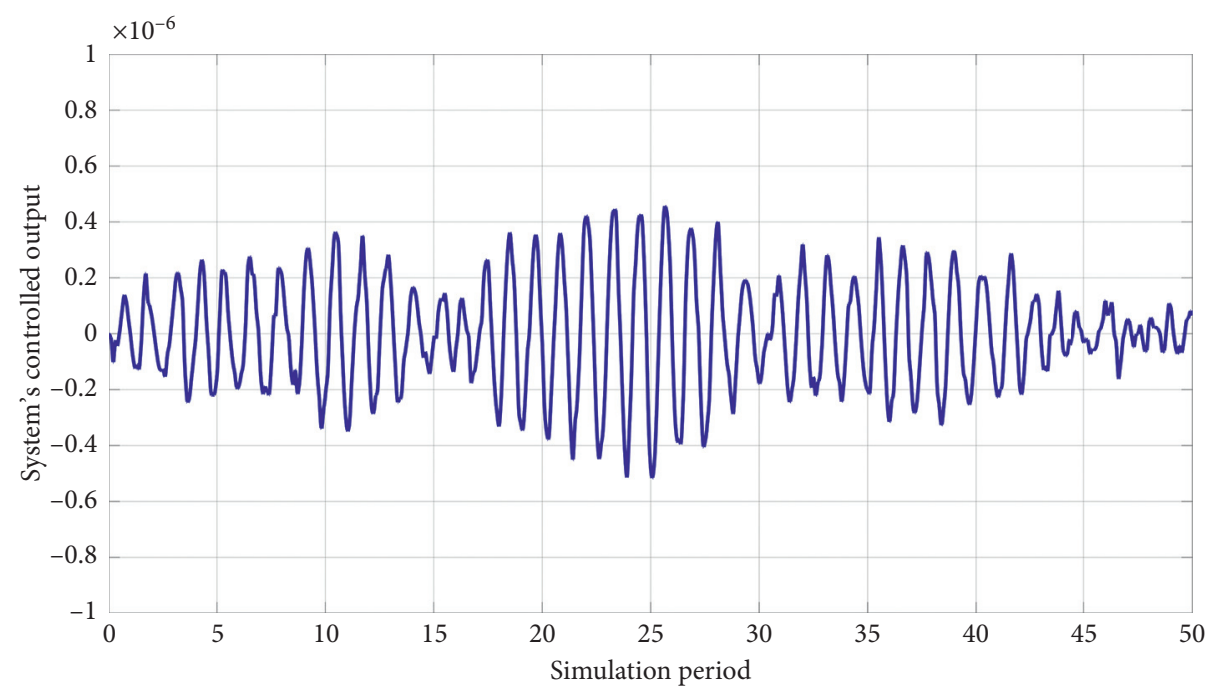

— System's controlled output (RPLNN)

FIGURE 9: Noise cancellation utilizing the RPLNN controller.

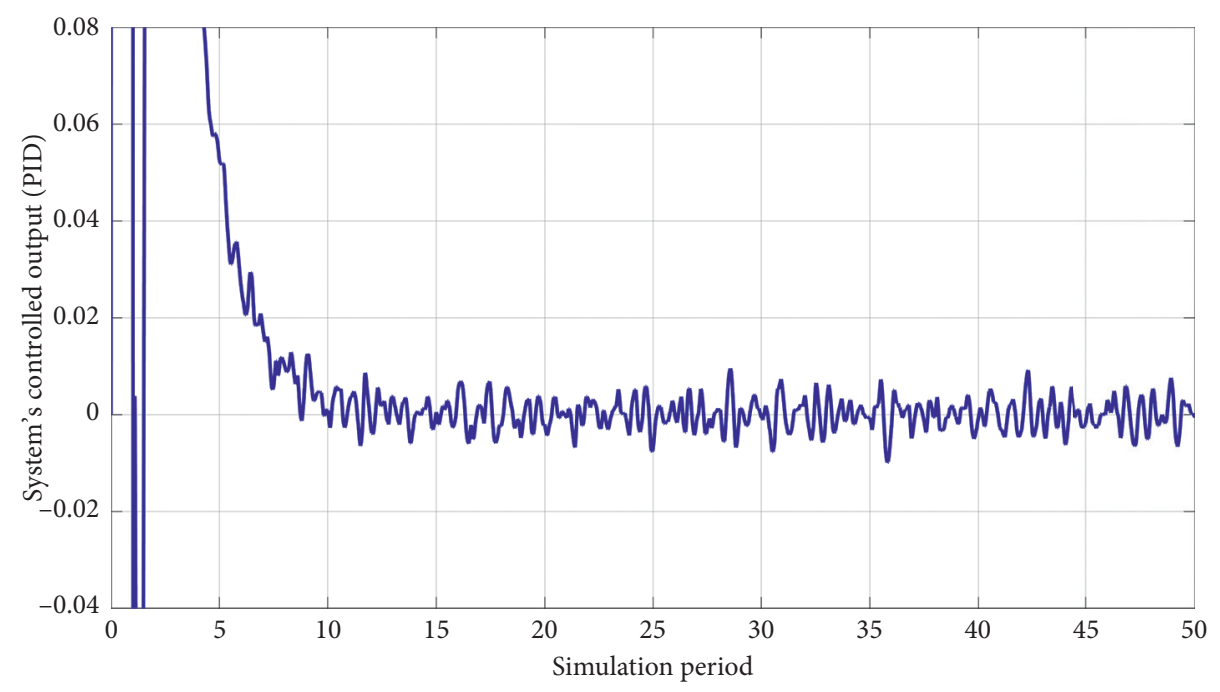

— System's controlled output (PID)

FIgURe 10: Noise cancellation utilizing PID controller.

controller has not shown an overshoot response, so it has a superior performance than the PID controller.

To have a better overview on the performance of the proposed RPLNN controller, the behavior of the system, once equipped with PID and then with RPLNN controllers, has been observed and compared.

As seen in Figure 9, at the first stages of the control process, the system equipped with PID controller reacts to the ground excitation with overshoot controlled response, and after three seconds starts to cancel the noise properly. However,as seen in Figure 10, by adopting the RPLNN controller system from the beginning of the control process shows a smooth response and prepares a convenient and safe ride for passengers.

Finally, it can be concluded that utilizing RPLNN controller, due to decreasing the imposed fluctuations to the vehicle from the road profile (which has been considered as Gaussian white noise), is an effective solution to be considered as noise cancelation tool, also it is obvious that, due to the reduction of vertical vibrations, the lifetime of the car parts has been increased.

\section{Conclusion and Future Work}

In the current research, to reduce the imposed vibrations to the quarter car model via the ground excitation an effective artificial intelligence controller for the active suspension system has been designed and modeled. The RPLNN algorithm has been utilized to design the proposed controller, and the road input signal has been modelled by utilizing Gaussian white noise. The proposed setup has been modelled by MATLAB and Simulink. The results show that the 
proposed RPLNN controller is able to reduce the undesired effect of the road condition on the vehicle, which ultimately results in decreasing the fuel consumption and increasing the lifetime of the parts of the vehicle. The performance of the RPLNN controller has been compared with the PID controller and results show that the RPLNN controller has a superior performance. As future work, other AI algorithms such as Fuzzy Logic and Neural Networks can be utilized as controllers, also adopting nonlinear models to evaluate the performance of the designed controller will be an alternative important topic to be investigated.

\section{Data Availability}

The related data are presented within the manuscript.

\section{Conflicts of Interest}

The authors declare they have no conflicts of interest.

\section{Acknowledgments}

This study was funded by the Research Council of Oman under Grant no. ORG/CBS/14/008.

\section{References}

[1] J. Palmer and S. Sljivar, Vehicle Fuel Consumption Monitor and Feedback Systems, Google Patents, Long Beach CA, USA, 2017.

[2] A. Azizi and P. G. Yazdi, "Introduction to fuel consumption optimization techniques," in Computer-Based Analysis of the Stochastic Stability of Mechanical Structures Driven by White and Colored Noise, pp. 1-12, Springer, Berlin, Germany, 2019.

[3] H. M. Ismail, H. K. Ng, C. W. Queck, and S. Gan, "Artificial neural networks modelling of engine-out responses for a light-duty diesel engine fuelled with biodiesel blends," Applied Energy, vol. 92, pp. 769-777, 2012.

[4] Y. Çay, A. Çiçek, F. Kara, and S. Sağiroğlu, "Prediction of engine performance for an alternative fuel using artificial neural network," Applied Thermal Engineering, vol. 37, pp. 217-225, 2012.

[5] S. Roy, R. Banerjee, A. K. Das, and P. K. Bose, "Development of an ANN based system identification tool to estimate the performance-emission characteristics of a CRDI assisted CNG dual fuel diesel engine," Journal of Natural Gas Science and Engineering, vol. 21, pp. 147-158, 2014.

[6] K. Muralidharan and D. Vasudevan, "Applications of artificial neural networks in prediction of performance, emission and combustion characteristics of variable compression ratio engine fuelled with waste cooking oil biodiesel," Journal of the Brazilian Society of Mechanical Sciences and Engineering, vol. 37, no. 3, pp. 915-928, 2015.

[7] A. C. Olivera, J. M. García-Nieto, and E. Alba, "Reducing vehicle emissions and fuel consumption in the city by using particle swarm optimization," Applied Intelligence, vol. 42, no. 3, pp. 389-405, 2015.

[8] S. Lotfan, R. A. Ghiasi, M. Fallah, and M. H. Sadeghi, "ANNbased modeling and reducing dual-fuel engine's challenging emissions by multi-objective evolutionary algorithm NSGAII," Applied Energy, vol. 175, pp. 91-99, 2016.

[9] F. Perrotta, T. Parry, and L. C. Neves, "Application of machine learning for fuel consumption modelling of trucks," in
Proceedings of the Big Data (Big Data) IEEE International Conference on, 2017, IEEE, Boston, MA, USA, pp. 3810-3815, December 2017.

[10] A. Azizi, "Computer-based analysis of the stochastic stability of mechanical structures driven by white and colored noise," Sustainability, vol. 10, no. 10, p. 3419, 2018.

[11] A. Azizi and P. G. Yazdi, "Introduction to noise and its applications," in Computer-Based Analysis of the Stochastic Stability of Mechanical Structures Driven by White and Colored Noise, pp. 13-23, Springer, Berlin, Germany, 2019.

[12] A. Azizi and P. G. Yazdi, "Noise control techniques," in Computer-Based Analysis of the Stochastic Stability of Mechanical Structures Driven by White and Colored Noise, pp. 61-73, Springer, Berlin, Germany, 2019.

[13] A. Azizi, "Hybrid artificial intelligence optimization technique," in Applications of Artificial Intelligence Techniques in Industry 4.0, pp. 27-47, Springer, Berlin, Germany, 2019.

[14] A. Azizi, "Introducing a novel hybrid artificial intelligence algorithm to optimize network of industrial applications in modern manufacturing," Complexity, vol. 2017, Article ID 8728209, 18 pages, 2017.

[15] A. Azizi, A. Barenji, R. Barenji, and M. Hashemipour, "Modeling mechanical properties of FSW thick pure copper plates and optimizing it utilizing artificial intelligence techniques," Sensor Netw Data Commun, vol. 5, no. 142, p. 2, 2016.

[16] A. Azizi, Applications of Artificial Intelligence Techniques in Industry 4.0, Springer, Berlin, Germany, 2018.

[17] Y. Zhang, K. Guo, D. Wang, C. Chen, and X. Li, "Energy conversion mechanism and regenerative potential of vehicle suspensions," Energy, vol. 119, pp. 961-970, 2017.

[18] I. Maciejewski, T. Krzyzynski, and H. Meyer, "Modeling and vibration control of an active horizontal seat suspension with pneumatic muscles," Journal of Vibration and Control, vol. 24, pp. 5938-5950, 2018.

[19] A. Azizi and P. G. Yazdi, "Mechanical structures: mathematical modeling," in Computer-Based Analysis of the Stochastic Stability of Mechanical Structures Driven by White and Colored Noise, pp. 37-59, Springer, Berlin, Germany, 2019.

[20] D. J. Inman, Vibration with Control, John Wiley \& Sons, Hoboken, NJ, USA, 2017.

[21] A. Azizi and P. G. Yazdi, "White noise: applications and mathematical modeling," in Computer-Based Analysis of the Stochastic Stability of Mechanical Structures Driven by White and Colored Noise, pp. 25-36, Springer, Berlin, Germany, 2019.

[22] A. Azizi, A. Vatankhah Barenji, and M. Hashmipour, "Optimizing radio frequency identification network planning through ring probabilistic logic neurons," Advances in Mechanical Engineering, vol. 8, no. 8, p. 1687814016663476, 2016.

[23] M. B. Menhaj and N. Seifipour, "Function optimization by RPLNN," in Proceedings of the 2002 International Joint Conference on Neural Networks, pp. 1522-1527, Honolulu, HI, USA, May 2002.

[24] A. Azizi, "Modern manufacturing," in Applications of Artificial Intelligence Techniques in Industry 4.0, pp. 7-17, Springer, Berlin, Germany, 2019.

[25] A. Azizi, "RFID network planning," in Applications of Artificial Intelligence Techniques in Industry 4.0, pp. 19-25, Springer, Berlin, Germany, 2019.

[26] A. Azizi and P. G. Yazdi, "Modeling and control of the effect of the noise on the mechanical structures," in Computer-Based Analysis of the Stochastic Stability of Mechanical Structures Driven by White and Colored Noise, pp. 75-93, Springer, Berlin, Germany, 2019. 